\title{
Risk of cardiovascular disease outcomes in primary care subjects with familial hypercholesterolaemia: A cohort study
}

\author{
Barbara Iyen $^{\mathrm{a}, *}$, Nadeem Qureshi ${ }^{\mathrm{a}}$, Joe Kai ${ }^{\mathrm{a}}$, Ralph K. Akyea ${ }^{\mathrm{a}}$, Jo Leonardi-Bee ${ }^{\mathrm{b}}$, Paul Roderick ${ }^{\mathrm{c}}$, \\ Steve E. Humphries ${ }^{\mathrm{d}, 1}$, Stephen Weng $^{\mathrm{a}, 1}$
}

a Primary Care Stratified Medicine Group, Division of Primary Care, University of Nottingham, UK

${ }^{\mathrm{b}}$ Division of Epidemiology and Public Health, University of Nottingham, UK

${ }^{\mathrm{c}}$ Faculty of Medicine, Primary Care and Population Sciences, University of Southampton, UK

${ }^{\mathrm{d}}$ Cardiovascular Genetics, Institute of Cardiovascular Science, University College London, UK

\section{H I G H L I G H T S}

- Subjects with clinical FH had elevated risks of stroke/TIA and PVD in addition to theraised risk of coronary heart disease.

- Undiagnosed FH subjects had much greater risks of all CVD outcomes than subjects with clinical FH diagnosis.

- Only $75 \%$ of the FH subjects were on lipid-lowering treatment, and only $38 \%$ of those on treatment were on high-potency statins.

\section{A R T I C L E I N F O}

\section{Keywords:}

Familial hypercholesterolaemia

Cardiovascular disease

Coronary heart disease

Stroke

Peripheral vascular disease

Epidemiology

\begin{abstract}
A B S T R A C T
Background and aims: Familial hypercholesterolaemia (FH) is a known major cause of premature heart disease. However, the risks of atherosclerotic disease in other vascular regions are less known. We determined the risk of major cardiovascular disease (CVD) outcomes associated with clinical FH.

Methods: In a retrospective cohort study (1 January, 1999 to 22 July, 2016), we randomly-matched 14,097 UK subjects with clinical FH diagnoses or characteristics (Simon-Broome definite or Dutch Lipid Clinic Score $>8$ ) to 42,506 subjects without FH by age, sex, general practice. We excluded those with CVD at baseline. Incident rates for coronary heart disease (CHD), stroke or transient ischaemic attack (TIA) and peripheral vascular disease (PVD) were estimated. Cox proportional hazards regression, stratified on matched-pairs, determined adjusted hazards ratios (HR) for incident CVD.

Results: During follow-up (median 13.8 years), incidence rates ( $95 \% \mathrm{CI}$ ) of CVD (per 1000 person-years) were 25.6 (24.8-26.3) in FH and 2.9 (2.8-3.1) in non-FH subjects. The risk of CHD, stroke/TIA and PVD was higher in FH compared to non-FH subjects: CHD (HR 10.63, 95\% CI 9.82-11.49), stroke/TIA (HR 6.74, 95\% CI 5.84-7.77), PVD (HR 7.17, 95\% CI 6.08-8.46). The risk of CVD was greater in those with FH characteristics (HR 13.52, 95\% CI 12.48-14.65) than those with clinical diagnoses (HR 1.66, 95\% CI 1.42-1.93).

Conclusions: In addition to the recognised increased risk of CHD, subjects with FH have greatly elevated risk of stroke/TIA and PVD. This emphasises need for early diagnosis and preventive interventions beyond CHD, to reduce CVD risk in these individuals.
\end{abstract}

\section{Introduction}

Familial hypercholesterolemia (FH) is a common monogenic autosomal dominant disorder causing raised low-density lipoprotein (LDL) cholesterol from birth. It affects up to 250,000 individuals in the UK, with an estimated prevalence of $1 / 270$ individuals in the general population with the heterozygous form [1-3]. Untreated FH is associated with a substantially higher risk of coronary heart disease (CHD) and premature death [4], but lipid-lowering therapy improves prognosis $[5,6]$, reducing risk of coronary heart disease and all-cause mortality by at least $44 \%$ in patients with heterozygous $\mathrm{FH}$ treated with moderate-to high-intensity statins [5-8].

\footnotetext{
* Corresponding author. Division of Primary Care, University of Nottingham, Room 1402, Tower Building, UK.

E-mail address: barbara.iyen@nottingham.ac.uk (B. Iyen).

${ }^{1}$ These authors contributed equally to this work.
} 
In England, the National Institute for Health and Care Excellence (NICE) guidelines, updated in 2017 [8], recommend that individuals with raised cholesterol levels in primary care are assessed for a clinical diagnosis of FH using Simon-Broome, Dutch Lipid Clinic Network (DLCN), or cholesterol above $>$ 99th percentile. Following a clinical FH diagnosis in primary care, these individuals should be referred for lipid specialist diagnosis, management and screening relatives. The FH diagnosis can be recorded in UK primary care electronic health records (EHRs) using specific disease codes, following specialist referral and diagnosis. Moreover, there are also undiagnosed patients in primary care with clinical characteristics (raised cholesterol, personal or family history of CHD, or clinical signs) who have the phenotype for FH.

While the association of $\mathrm{FH}$ with premature CHD is well known, the risk of atherosclerotic disease in other vascular regions among subjects with FH is less clear, and evidence from previous studies is conflicting [9-12]. This study sought to determine the cardiovascular disease risk profile of subjects with FH in the general population using longitudinal data from patients' primary care electronic health records. We assessed the incidence and risks of CHD, stroke/transient ischaemic attacks and peripheral vascular disease in subjects in primary care with clinical FH.

\section{Materials and methods}

\subsection{Study design and data source}

We conducted a retrospective matched cohort study using data from the Clinical Practice Research Datalink (CPRD). The CPRD is a large nationally representative electronic database of anonymised primary care data of subjects in the UK. It includes data from over 20 million patient lives with over 5 million patients currently registered and active. The database contains information on patient characteristics, symptoms, clinical diagnoses, laboratory test results, medication prescriptions and referrals to secondary care [13]. Access to the data and ethical approval was granted by the CPRD Independent Scientific Advisory Committee (Protocol number 16_191R2).

\subsection{Study population}

We identified all adults with FH in primary care during the study period: 1 January 1999 to 22 July 2016. This study cohort comprised subjects who had at least one cholesterol measurement (total cholesterol or low-density lipoprotein cholesterol) during the study period with either:

\section{- a documented FH diagnosis, or}

- the clinical phenotype of definite FH using the Simon Broome (SB) or Dutch Lipid Clinic Network (DLCN) criteria [1].

Subjects' follow-up started only after they had contributed one year of records in their general practice. Subjects who had disease (Read) codes suggesting pre-existing CVD (coronary heart disease, stroke, transient ischaemic attack or peripheral vascular disease) prior to the start of their follow-up were excluded.

Each subject with FH was individually matched to three control subjects without FH, by 5-year age-bands, sex and general practice to eliminate confounding by these variables. Eligibility criteria for subjects without FH were: cholesterol testing done within six months of the follow-up start date of their matched FH case, no documented diagnosis of FH and no pre-existing CVD.

All subjects were followed up until their first diagnosis of cardiovascular disease. Subjects who did not develop CVD were followed up until date of death, transfer out of the practice or study end date, whichever occurred first.

\subsection{Covariates}

All analyses were stratified on the matched pairs and therefore controlled for any confounding effect of age, sex and general practice. We collected data on other demographic variables such as ethnicity, lifestyle, medication and comorbidities that could potentially confound the relationship between FH and future CVD in this cohort. Included in the analyses were, baseline records of smoking status, history of alcohol misuse, obesity/overweight, hypertension, type 1 diabetes (T1DM), type 2 diabetes (T2DM), rheumatoid arthritis and other inflammatory/ immunological conditions, chronic kidney disease, atrial fibrillation, treatment with lipid-reducing medication, potency of prescribed statins, use of antipsychotics, corticosteroid use and family history of premature CHD. We also adjusted for any effects of potential improvement in FH management on the risk of CVD, due to the publication of NICE guidelines, by indicating whether follow-up started before or on/after 2008 for subjects in the cohort.

\subsection{Outcome ascertainment}

Incident cardiovascular disease was defined as any new clinical diagnosis of coronary heart disease, stroke/transient ischaemic attack (TIA) or peripheral vascular disease. These were identified from subjects' primary care EHRs during the study period, as were mortality and date of death. Disease codes used for cardiovascular disease are shown in the supplementary online files.

\subsection{Sample size calculation}

Based on findings from previous research [14], we estimated a minimum hazard ratio of 1.2 for overall atherosclerotic CVD risk. To achieve this, a minimum total cohort size of 9450 individuals was required with an expectation of 1265 CVD events at $90 \%$ power and significance level of 0.05 (two-sided test of significance). Matching one FH subject to three controls allowed for optimum statistical power for the analysis based on previous recommendations [15].

\subsection{Statistical analyses}

Baseline descriptive analyses were performed for all subjects in the cohort and results were represented as number (\%), mean (SD) and median (IQR) for categorical, normal continuous and non-normal continuous variables respectively. Missing variables were presented in result tables. Appropriate statistical tests such as chi-squared, t-tests and analyses of variance tests (ANOVA) were used to assess differences between the groups of interest. The incidence rates of CVD were determined for FH and non-FH groups, presented per 1000-person years at risk. Cox proportional hazards regression was used to derive hazards ratios for first onset of any CVD and secondarily, hazards ratios for the various major CVD subtypes - CHD, stroke/TIA and peripheral vascular disease, for subjects with FH compared to those without FH. Analyses were stratified on the matched variables and also adjusted for individuals' demographics, lifestyle factors, comorbidities and prescribed medication use as listed above. Confounder selection used the changein-estimate criteria [16], and any covariate which changed the effect size of the univariate exposure-outcome model by $10 \%$ was considered an important confounder and included in the fully-adjusted model. Statistical tests of the proportional hazards assumption found the Cox proportional hazards regression to be suitable for the analyses. In subjects with missing or unrecorded categorical clinical variables, the common assumption was made that these individuals did not have the condition. Multiple imputation, the standard epidemiological approach used in research studies to provide unbiased estimates for missing values when data are assumed to be missing at random [17], was used to estimate missing BMI data. Complete case analyses was adopted in the analyses of other continuous variables with missing data, such as 
Triglycerides. All analyses were performed using STATA SE15.

\subsection{Sensitivity analyses}

Since lower levels of triglycerides are associated with higher rates of detection of $\mathrm{FH}$ mutation in subjects with a clinical diagnosis of $\mathrm{FH}$ $[18,19]$, we evaluated the effect of limiting analyses to a subset of those with stringent lipid criteria for $\mathrm{FH}$. We also assessed the impact of $\mathrm{FH}$ diagnosis on CVD risk by performing separate analyses on subjects with recorded diagnoses of $\mathrm{FH}$ and those undiagnosed subjects with the clinical phenotype (Simon-Broome definite or DLC Score $>8$ ).

\section{Results}

\subsection{Cohort characteristics}

There were 3,936,934 subjects in CPRD with records of either total or LDL-cholesterol measurement between 1 January 1999 and 22 July 2016. Among these, 14,097 subjects had clinical FH and no prior history of cardiovascular disease at baseline. This comprised 5152 subjects with documented diagnosis of FH in the EHR, and 8945 subjects who had no documented FH diagnosis but had the clinical phenotype of $\mathrm{FH}$ based on SB or DLCN diagnostic criteria for definite FH. Of these identified subjects, $53.3 \%$ were females, the mean age at the start of follow-up was 42 years, and mean body-mass index was $27.3 \mathrm{~kg} / \mathrm{m}^{2}$. FH subjects were matched with 42,506 non-FH subjects. As individuals with and without FH were matched on age and sex, the distribution of these characteristics was similar in both groups. The median follow-up time for FH and non-FH subjects was 13.8 years (IQR 8.4-17.7 years) and the average follow-up in person-years for subjects with and without FH was 174,950 and 588,470 person-years, respectively.
The baseline demographic and clinical characteristics of subjects with and without FH are shown in Table 1. As expected, there was a significantly higher proportion of FH subjects with a family history of premature coronary heart disease than non-FH subjects $(5.1 \%$ v. $2.7 \%$, $p<0.001$ ); and more FH than non-FH subjects were on lipid-lowering medication at the start of the study $(19.1 \%$ vs. $4.7 \%, p<0.001)$. Ethnicity records were missing in $85 \%$ of subjects, but in those where it was recorded, $80 \%$ were White Caucasian. BMI records were available for $40 \%$ of subjects, so multiple imputation with chained equations was used to estimate missing BMI values.

\subsection{Lipid characteristics of the cohort}

For subjects on statins or other cholesterol-lowering medications with known potency, the corrected levels of baseline cholesterol were estimated from observed levels, based on estimated percentage reduction in LDL-cholesterol with statins of different potencies [20]. As expected, the mean total cholesterol concentration was significantly higher in those with $\mathrm{FH}(9.30$ [SD 2.6] mmol/L) compared to those without FH (5.98 [SD 1.6] mmol/L), respectively. In FH and non-FH subjects, mean LDL-cholesterol at baseline was $5.72 \mathrm{mmol} / \mathrm{L}$ [SD 2.1] and $3.63 \mathrm{mmol} / \mathrm{L}$ [SD 1.1], respectively. Fig. 1 shows the distribution of baseline LDL-cholesterol among subjects with and without FH.

\subsection{Prescribing of statins and other lipid lowering treatments}

At the start of the study, $19.1 \%$ of subjects with FH and $4.7 \%$ of nonFH subjects were on lipid-lowering treatments. Over the duration of follow-up, the proportion of $\mathrm{FH}$ and non-FH subjects on lipid-lowering treatments increased to $75 \%$ and $20 \%$, respectively. There was a marginal increase in the proportion of non-FH subjects prescribed high

Table 1

Baseline demographic and clinical characteristics of subjects with clinical FH and non-FH subjects.

\begin{tabular}{|c|c|c|c|c|}
\hline Risk factor variable & Unit & FH subjects $n=14,097$ & Non-FH subjects $n=42,506$ & $p$-value \\
\hline Age at start of study & mean (SD) & $42.5(11.7)$ & $41.6(12.5)$ & \\
\hline \multicolumn{5}{|l|}{ Sex } \\
\hline Male & n $(\%)$ & $6578(46.7)$ & $19,843(46.7)$ & \\
\hline Female & & 7519 (53.3) & $22,663(53.3)$ & \\
\hline Follow-up (years) & median (IQR) & $12.4(7.1-16.8)$ & $14.2(8.8-17.8)$ & \\
\hline Body mass index & $\mathrm{kg} / \mathrm{m}^{\mathrm{b}}(\mathrm{SD})$ & $27.8(5.4)$ & $26.2(5.6)$ & $<0.001$ \\
\hline Alcohol misuse $^{\mathrm{a}}$ & n (\%) & 265 (1.9) & $712(1.7)$ & 0.106 \\
\hline Ever-smoked record ${ }^{\mathrm{b}}$ (yes/no) & n (\%) & $11,518(81.7)$ & 34,395 (80.9) & 0.038 \\
\hline Hypertension & n (\%) & $783(5.6)$ & $2393(5.6)$ & 0.736 \\
\hline Atrial fibrillation & n $(\%)$ & $35(0.3)$ & $120(0.3)$ & 0.503 \\
\hline Chronic kidney disease & n $(\%)$ & $57(0.4)$ & $127(0.3)$ & 0.056 \\
\hline Type 1 diabetes & n (\%) & $60(0.4)$ & $247(0.6)$ & 0.029 \\
\hline Type 2 diabetes & n $(\%)$ & $311(2.2)$ & $726(1.7)$ & $<0.001$ \\
\hline Overweight/obesity & n (\%) & $525(3.7)$ & $1307(3.1)$ & $<0.001$ \\
\hline Rheumatoid arthritis \& other inflammatory diseases & n (\%) & $100(0.7)$ & $285(0.7)$ & 0.626 \\
\hline Family history of coronary heart disease & $\mathrm{n}(\%)$ & $712(5.1)$ & $1156(2.7)$ & $<0.001$ \\
\hline HIV & n $(\%)$ & $6(0.04)$ & $16(0.04)$ & 0.797 \\
\hline Antipsychotic use & n (\%) & $491(3.5)$ & $1165(2.7)$ & $<0.001$ \\
\hline Oral corticosteroids & n $(\%)$ & $313(2.2)$ & $920(2.2)$ & 0.693 \\
\hline Immunosuppressant drugs & $\mathrm{n}(\%)$ & $315(2.2)$ & $926(2.2)$ & 0.694 \\
\hline Lipid-lowering treatment ${ }^{c}$ & n $(\%)$ & $2692(19.1)$ & $2007(4.7)$ & $<0.001$ \\
\hline \multicolumn{5}{|l|}{ Lipid profile $^{\mathrm{d}}(\mathrm{mmol} / \mathrm{L})$} \\
\hline Total cholesterol & mean $(S D)$ & $9.30(2.6)$ & $5.98(1.6)$ & \\
\hline LDL cholesterol & mean (SD) & $5.72(2.1)$ & $3.63(1.1)$ & \\
\hline Triglyceride & median (IQR) & $2.10(1.3-3.5)$ & $1.30(0.9-1.9)$ & \\
\hline \multicolumn{5}{|l|}{ Statin potency } \\
\hline Low & n (\%) & $57(0.4)$ & $49(0.1)$ & \\
\hline Medium & & $466(3.3)$ & $384(0.9)$ & \\
\hline High & & $447(3.2)$ & $145(0.3)$ & \\
\hline Non-statin lipid lowering medication & n (\%) & $1722(12.2)$ & $1429(3.4)$ & \\
\hline
\end{tabular}

a Numbers indicate proportion of subjects with record of alcohol misuse.

b Numbers indicate proportion of subjects with record of ever-smoking.

c Lipid-lowering treatment include statins, fibrates and combination therapy (statins and ezetimibe).

d Total cholesterol and LDL-C correction done for patients on cholesterol lowering medication with known potency (based on percentage reduction in LDL-C estimated in a previous study) (1). 


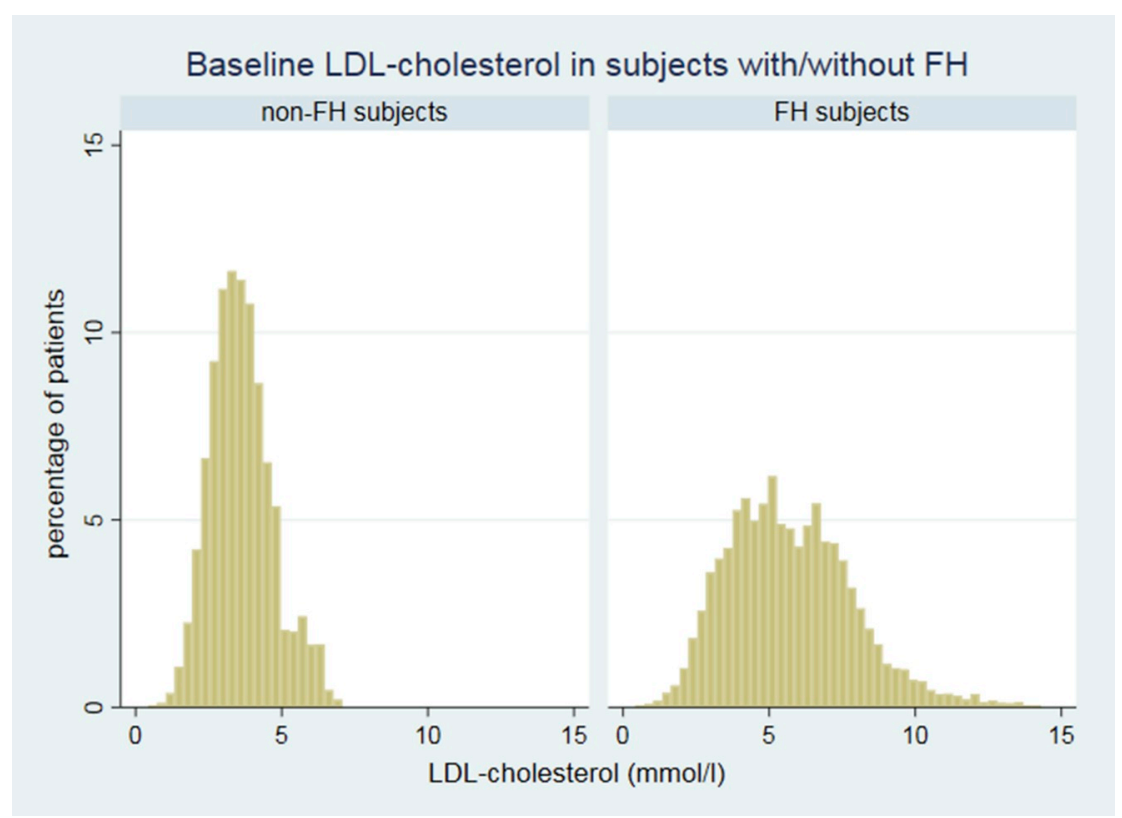

Fig. 1. Distribution of LDL-cholesterol levels (millimoles/litre) in FH and non-FH subjects.

Each bar's height represents the percentage of subjects in that category. Height of the bars are scaled so that the sum of heights equals 100.

Table 2

Proportion of subjects of lipid-lowering therapy of different potencies.

\begin{tabular}{|c|c|c|c|c|c|c|c|c|c|c|}
\hline \multirow{2}{*}{ Study period } & \multicolumn{2}{|c|}{ Low potency statin (\%) } & \multicolumn{2}{|c|}{ Medium potency statin (\%) } & \multicolumn{2}{|c|}{ High potency statin (\%) } & \multicolumn{2}{|c|}{ Other LLT (\%) } & \multicolumn{2}{|c|}{ No LLT (\%) } \\
\hline & FH & Non FH & FH & Non FH & FH & Non FH & FH & Non FH & FH & Non FH \\
\hline Start of study & 0.40 & 0.10 & 3.30 & 0.90 & 3.20 & 0.30 & 12.20 & 3.40 & 80.9 & 94.3 \\
\hline Study follow-up & 1.98 & 1.22 & 35.61 & 14.06 & 37.54 & 4.60 & 0.41 & 0.27 & 24.46 & 79.86 \\
\hline
\end{tabular}

intensity statins, from $0.3 \%$ at baseline to $4.6 \%$ during follow-up. This contrasts with a greater increase in high intensity statins prescribed in FH subjects from $3.2 \%$ at baseline to $37.5 \%$ during follow-up. Table 2 shows the proportion of $\mathrm{FH}$ and non-FH subjects treated with lipidlowering medication of different potencies, at baseline and during the period of follow-up (also shown in Supplemental Fig. 2).

\subsection{Cardiovascular disease outcomes}

There were a total of 6202 incident cases of cardiovascular disease (CHD, stroke, TIA or PVD) during follow-up. These were identified in $31.7 \%$ of individuals with $\mathrm{FH}$ and $4.1 \%$ of non-FH individuals. Clinical risk factors for CVD such as hypertension, type 2 diabetes, higher body mass index and male sex were more prevalent in FH subjects who were diagnosed with CVD than those who remained free of CVD during follow-up. The prevalence of CKD, atrial fibrillation and baseline LDLcholesterol levels did not differ significantly between the FH subjects who developed CVD and those who did not. Unexpectedly, smoking prevalence was significantly higher in FH subjects who did not develop CVD compared to those who did (Supplemental Table A).

Overall, the incidence rates of CVD among $\mathrm{FH}$ and non-FH individuals (per 1000 person-years at risk) were 25.6 and 2.9, respectively. The incidence rate in $\mathrm{FH}$ compared to non-FH individuals was highest for CHD (incidence rate 20.3 versus 2.0), with incidence rates of stroke/TIA and PVD also higher among those with FH compared to nonFH individuals (Table 3). The overall mean age at first diagnosis of CHD, stroke/TIA and PVD was 53.3 years, 56 years and 55.5 years, respectively. CVD outcomes were diagnosed approximately 10 years earlier in those with FH compared to those without FH.

\subsection{Hazards ratio of cardiovascular disease}

Table 4 shows the number of CVD events and hazards ratios for all CVD subtypes among FH and non-FH subjects. FH subjects were more likely to have incident CVD than non-FH subjects (hazards ratio [HR] 9.14, 95\% CI 8.55-9.76, $p<0.001$ ). This comprised higher risk of incident CHD (HR 10.63, 95\% CI 9.82-11.49, $p<0.001$ ), stroke/TIA (HR 6.74, 95\% CI 5.84-7.77, $p<0.001$ ) and peripheral vascular disease (HR 7.17, 95\% CI 6.08-8.46, $p<0.001$ ). Adjustment for demographic factors, clinical covariates, LDL-cholesterol levels and the effect of the 2008 NICE guidelines resulted in no major change in these hazard ratios ( $<10 \%$ change-in-estimate criteria).

Stratifying the analysis between FH and various CVD outcomes by sex, demonstrated a statistically significant higher risk of all the different CVD endpoints within sexes $(p<0.001$ for all endpoints in males and females). Compared to subjects of the same sex, the higher CHD risk among males with FH was markedly higher than the higher risk among females with FH (Fig. 2). Between-sex comparisons showed statistically higher hazards ratio for all CVD outcomes associated with $\mathrm{FH}$, in males compared with females $(p<0.001$ for all CVD endpoints).

\subsection{Sensitivity analyses}

On restricting analyses to a subset of FH subjects with total cholesterol $>7.5 \mathrm{~mm} / 1$ and triglycerides $<3.5 \mathrm{mmol} / \mathrm{L}$, the hazards ratios for all CVD outcomes including stroke/TIA and peripheral vascular disease remained substantially higher in subjects with FH compared to those without FH (Table 5). The hazards ratio estimates for these outcomes were, however, lower than estimates from the primary analyses. 
Table 3

Incidence rate of CVD outcomes in $\mathrm{FH}$ and non-FH subjects.

\begin{tabular}{|c|c|c|c|c|c|c|c|}
\hline \multirow{2}{*}{ CVD outcome } & \multicolumn{2}{|c|}{ Age in years at first CVD event (mean (SD)) } & \multicolumn{2}{|c|}{ Number of new events } & \multicolumn{2}{|c|}{ Rate per 1000 person-years $(95 \% \mathrm{CI})$} & \multirow[t]{2}{*}{$p$-value for CVD rate ratio } \\
\hline & FH subjects & Non-FH subjects & FH subjects & Non-FH subjects & FH subjects & Non-FH subjects & \\
\hline Any cardiovascular disease & & & 4474 & 1728 & $25.6(24.8-26.3)$ & $2.9(2.8-3.1)$ & $<0.0001$ \\
\hline Coronary heart disease & $50.7(7.5)$ & $61.1(10.9)$ & 3545 & 1173 & $20.3(19.6-20.9)$ & $2.0(1.9-2.1)$ & $<0.0001$ \\
\hline Stroke/TIA & $51.9(8.5)$ & $63.7(11.2)$ & 764 & 405 & $4.3(4.1-4.7)$ & $0.7(0.6-0.8)$ & $<0.0001$ \\
\hline Peripheral vascular disease & $52.4(8.2)$ & $61.7(10.9)$ & 592 & 295 & $3.4(3.1-3.7)$ & $0.5(0.4-0.6)$ & $<0.0001$ \\
\hline
\end{tabular}

Person-time at risk for subjects with FH (1000 person-years): 174.95.

Person-time at risk for non-FH subjects (1000 person-years): 588.47.

Table 4

Hazards ratios for CVD outcomes among subjects identified with FH.

\begin{tabular}{|c|c|c|c|c|}
\hline CVD outcome & $\begin{array}{l}\text { Total n (\%) } \\
N=56,603\end{array}$ & FH n (\%) n $=14,097$ & Non-FH n (\%) $\mathrm{n}=42,506$ & $\mathrm{HR}^{\mathrm{a}}$ for CVD $(95 \% \mathrm{CI})$ \\
\hline All CVD outcomes & $6202(11.0)$ & 4474 (31.7) & $1728(4.1)$ & $9.14(8.55-9.76)$ \\
\hline Coronary heart disease & $4718(8.3)$ & 3545 (25.2) & $1173(2.8)$ & $10.63(9.82-11.49)$ \\
\hline Stroke/TIA & $1169(2.1)$ & $764(5.4)$ & $405(1.0)$ & $6.74(5.84-7.77)$ \\
\hline Peripheral vascular $\mathrm{dx}$ & $887(1.6)$ & $592(4.2)$ & $295(0.7)$ & $7.17(6.08-8.46)$ \\
\hline
\end{tabular}

${ }^{\text {a }}$ Hazards ratios derived using Cox regression models stratified on matched pairs, with matching done on age, sex and general practice.

Further restriction to a smaller subgroup of FH subjects with triglycerides 2.16 or less, resulted in even lower hazards ratio estimates for CVD than the subgroup with triglycerides $<3.5 \mathrm{mmol} / \mathrm{L}$. There also remained higher risk of all CVD outcomes in this subgroup of FH subjects compared to non-FH subjects (Supplemental Table B).

Separate analyses of CVD outcomes in diagnosed FH subjects and those with FH characteristics who were undiagnosed, showed a higher risk of CHD in both groups of patients. Compared to subjects without $\mathrm{FH}$, subjects with undiagnosed FH had markedly elevated hazards ratio for CHD (HR 15.32, 95\% CI 13.91-16.88, $p<0.0001$ ) as well as significantly elevated risk of other CVD subtypes such as stroke/TIA and PVD (Table 6). In patients with documented FH diagnoses, there was also a significant but marginally higher risk of CHD compared to nonFH subjects (HR 2.05, 95\% CI 1.72-2.45, $p<0.0001$ ). There was no statistically significant difference in the risk of stroke/TIA and PVD among diagnosed FH subjects compared to non-FH subjects. At the start of the study, lipid lowering treatments were prescribed in $12.2 \%$ of undiagnosed subjects with $\mathrm{FH}$ phenotype and $31.0 \%$ of diagnosed $\mathrm{FH}$ subjects. During follow-up, the proportion on treatment increased to $74.4 \%$ and $77.6 \%$, respectively.

\section{Discussion}

\subsection{Summary of principal findings}

This large cohort study has found subjects with clinical FH have significantly increased risks of all major types of CVD, which occurred at a younger age, compared with age- and sex-matched subjects without FH. Diagnosed FH subjects had a two-fold higher risk of CHD than subjects without FH. Subjects with undiagnosed FH had a 15-fold higher risk of CHD as well as higher risks of stroke/TIA and PVD. Using stringent lipid thresholds (elevated cholesterol/low triglycerides) to determine FH phenotypes still maintained elevated risk of CVD in FH subjects.

\subsection{Comparison with existing literature}

Few studies have assessed the risk of CVD outcomes in primary care subjects with a clinical diagnosis of FH. Similar to our study, elevated risks of CHD and total atherosclerotic CVD were demonstrated in a Spanish multi-centre prospective cohort study of molecularly defined

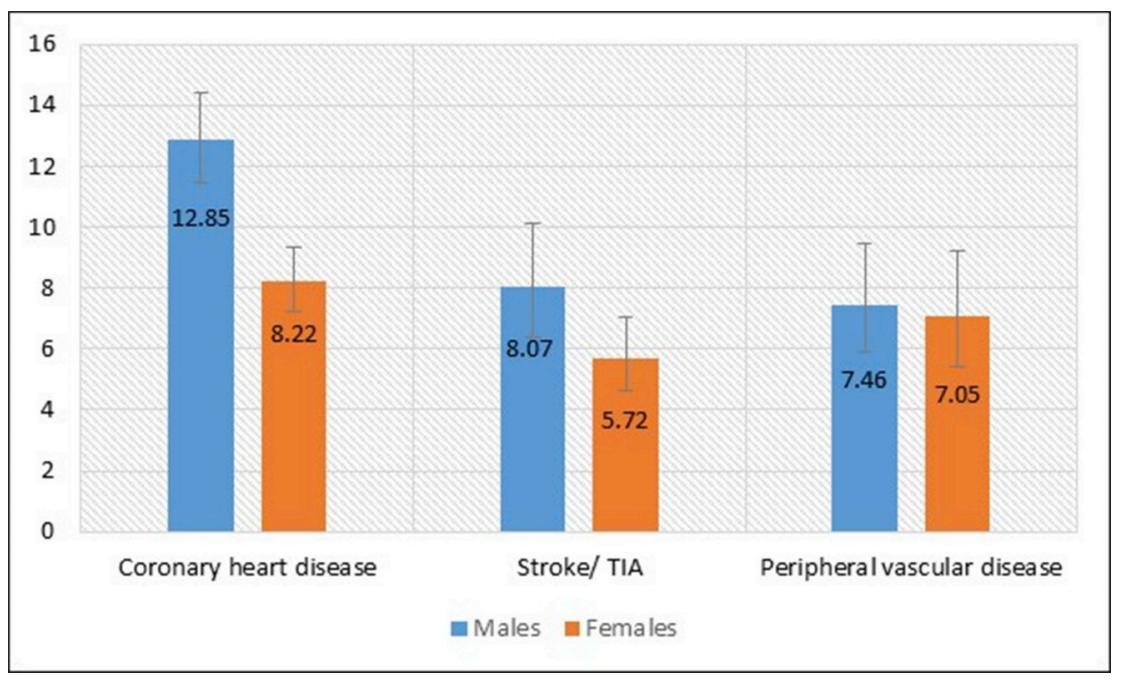

Fig. 2. Hazards ratio for CVD outcomes in subjects with FH, stratified by sex.

Each bar represents the hazard ratio for the CVD subtype among FH and non-FH subjects of the same sex. The error bars represent the $95 \%$ confidence intervals. All differences between males and females were statistically significant with $p<0.001$ for all endpoints. 
Table 5

Hazards ratios for CVD outcomes among the subgroup of $\mathrm{FH}$ subjects with total cholesterol ${ }^{\mathrm{b}}>7.5$ and triglycerides $<3.5$.

\begin{tabular}{|c|c|c|c|c|}
\hline CVD outcome & $\begin{array}{l}\text { Total n (\%) } \\
\mathrm{N}=23,027\end{array}$ & FH n (\%) n $=6318$ & Non-FH $n(\%) n=16,709$ & $\mathrm{HR}^{\mathrm{a}}$ for CVD $(95 \% \mathrm{CI})$ \\
\hline All CVD outcomes & 2501 (10.9) & $1703(27.0)$ & $798(4.8)$ & $6.19(5.62-6.83)$ \\
\hline Coronary heart disease & $1884(8.2)$ & $1352(21.4)$ & $532(3.2)$ & $7.29(6.49-8.19)$ \\
\hline Stroke/TIA & $503(2.2)$ & $298(4.7)$ & $205(1.2)$ & $4.42(3.60-5.45)$ \\
\hline Peripheral vascular $\mathrm{dx}$ & $346(1.5)$ & $218(3.5)$ & $128(0.8)$ & $5.02(3.91-6.45)$ \\
\hline
\end{tabular}

a Hazards ratios derived using Cox regression models stratified on matched pairs.

b Total cholesterol correction done for subjects on cholesterol lowering medication with known potency (based on estimated percentage reduction in LDL-C with statin potency [19].

FH patients [21] as well as a population-based study of patients with clinical FH in the United States [14]. These studies did not, however, evaluate the risk of PVD or TIA and stroke as separate entities. A more recent cohort study of FH registry patients in Norway also found an increased risk of CHD in subjects with genetically diagnosed $\mathrm{FH}$, compared to the general population [22]. Similar to other registry-based researches [5,6], the excess CHD risk was highest in the youngest age group with an increase in the relative risk of fatal and non-fatal CHD before the age of 40 years, which decreases with increasing age [5,6,22].

Previous research has shown that the risk of a fatal coronary event associated with FH is greatest in the 20-39 year age group [23]. The mean age of subjects in our study was 42 years, and may partly be due to the fact that previously asymptomatic individuals become identified in primary care during the UK NHS health check, which is offered to adults aged between 40 and 74 years [24]. In addition, in patients less than 40 years, a clinical diagnosis of FH in primary care may only be recognised after the first diagnosis of CVD. We adjusted for the effect of age in the univariate association of FH with CVD but found no significant attenuation of the hazard ratio (not shown). As age did not change the hazard ratio by up to $10 \%$, it was not considered a significant confounder of the association of FH with CVD in our study.

We found that only $36 \%$ of the subjects with clinical $\mathrm{FH}$ had a documented diagnosis, and only $19 \%$ of all FH subjects were on lipidlowering treatment at baseline (comprising $12.2 \%$ of undiagnosed $\mathrm{FH}$ subjects and $31 \%$ of subjects with documented FH diagnosis). This is consistent with findings of underdiagnoses and suboptimal treatment of phenotypic $\mathrm{FH}$ patients in a European Mediterranean primary care population [25]. The much higher risk of CHD among undiagnosed FH subjects who were not optimally treated also corroborates findings from a Danish population-based study [26], which demonstrated a much higher $\mathrm{FH}$-associated risk of $\mathrm{CHD}$ in $\mathrm{FH}$ subjects not on cholesterol- reducing medication compared to those on treatment with cholesterolreducing medication.

Several studies assessing the risk of stroke in subjects with FH provide conflicting results $[9,10,12]$. A recent study found no association between genetically-confirmed $\mathrm{FH}$ and ischaemic stroke risk; however, clinical FH, according to DLCN criteria, was found to be associated with a 2.5 -fold higher risk of ischaemic stroke which may, perhaps, be attributed to the DLCN diagnostic criteria of personal premature CHD [10].

In patients with treated $\mathrm{FH}$ in the Simon Broome register, the risk of fatal stroke was not significantly increased when compared with the general population [9]. This may be due to regular monitoring of subjects in the register, resulting in the early identification and management of hypertension, and the consequent reduction of risk of stroke [10]. It was suggested by the Simon Broome study authors that the finding may be due to possible underestimation of stroke risk, with susceptible individuals dying from $\mathrm{CHD}$ at much earlier ages. With the current trend to reduction in MI and CHD mortality, there may be an increase in the incidence of other CVD events, like stroke and PVD.

A few small-scale studies in the pre-statin era demonstrated an association between FH and stroke. An increased risk of familial hyperlipidaemia was found in a small study of young patients with nonembolic ischaemic stroke of unknown aetiology [27]. Another study reported higher prevalence of carotid bifurcation atherosclerosis in patients with $\mathrm{FH}$ [28]. Ischaemic stroke risk was also shown to be 20 times higher in subjects with heterozygous FH than in the general Finnish population [12].

We found that FH subjects who developed CVD had a higher BMI as well as higher prevalence of hypertension and type 2 diabetes than $\mathrm{FH}$ subjects who did not develop CVD. This is consistent with findings from the Spanish SAFEHEART cohort study of patients with $\mathrm{FH}$, which showed that male sex, high blood pressure and increased BMI were

Table 6

Hazards ratios for CVD in GP-diagnosed FH and undiagnosed (phenotypic) FH subjects.

\begin{tabular}{|c|c|c|c|c|c|}
\hline & \multicolumn{5}{|c|}{ CVD outcome } \\
\hline \multicolumn{6}{|c|}{ Subjects with FH diagnosis } \\
\hline & $\mathrm{N}=20,796$ & $\mathrm{n}=5152$ & $\mathrm{n}=15,644$ & & \\
\hline All CVD outcomes & $883(4.3)$ & $301(5.8)$ & $582(3.7)$ & $1.66(1.42-1.93)$ & $<0.0001$ \\
\hline Coronary heart disease & $613(3.0)$ & $240(4.7)$ & $373(2.4)$ & $2.05(1.72-2.45)$ & $<0.0001$ \\
\hline Stroke/TIA & $186(0.9)$ & $37(0.7)$ & $149(1.0)$ & $0.77(0.52-1.14)$ & 0.188 \\
\hline Peripheral vascular $\mathrm{dx}$ & $141(0.7)$ & $41(0.8)$ & $100(0.6)$ & $1.47(0.99-2.19)$ & 0.056 \\
\hline \multicolumn{6}{|c|}{ Subjects with phenotype for FH but no diagnosis } \\
\hline & $\mathrm{N}=35,807$ & $\mathrm{n}=8945$ & $\mathrm{n}=26,862$ & & \\
\hline All CVD outcomes & 5319 (14.9) & $4173(46.7)$ & $1146(4.4)$ & $13.52(12.48-14.65)$ & $<0.0001$ \\
\hline Coronary heart disease & $3933(11.0)$ & $3167(35.4)$ & $766(2.9)$ & $15.32(13.91-16.88)$ & $<0.0001$ \\
\hline Stroke/TIA & $830(2.3)$ & $616(6.9)$ & $214(0.8)$ & $10.92(9.02-13.22)$ & $<0.0001$ \\
\hline Peripheral vascular $\mathrm{dx}$ & $556(1.6)$ & $390(4.4)$ & $166(0.6)$ & $8.47(6.80-10.54)$ & $<0.0001$ \\
\hline
\end{tabular}


independent predictors of atherosclerotic CVD in FH [21]. Similar findings of significant and independent association between clinical risk factors and CVD risk in individuals with FH was also demonstrated in a systematic review [29]. Interestingly, unlike the SAFEHEART study, our study findings did not demonstrate a positive association between smoking and CVD in subjects with FH and we found no significant difference in baseline LDL-cholesterol levels in FH subjects who developed CVD compared to those who did not.

\subsection{Strengths and limitations}

To our knowledge, this is the first study to identify elevated risk of the diverse range of CVD outcomes in patients with clinical diagnoses of FH in a real-world clinical setting. The use of an established and quality assured database of comprehensively coded clinical data from subjects' primary care records [13] has allowed us to robustly investigate the associations between FH and CVD outcomes using a large sample size and prospective longitudinal design, minimising selection or recall bias.

We identified subjects with clinical FH using specific disease coding for $\mathrm{FH}$ as well as clinical phenotype based on standard diagnostic criteria (Simon Broome and Dutch Lipid Clinic Network). This study cohort represents clinical FH subjects found in the general clinical population setting and so, the CVD risk estimates from this large study can be confidently generalised to subjects identified with clinical FH in 'real world' primary care practice. Further, the highly elevated risk of CHD found in the study is entirely consistent with that found in specialist settings and disease registers, thereby underlining the validity of the clinical FH cohort identified here, and the applicability of new findings for risk of stroke and PVD.

The FH mutation-detection rate among patients with clinical $\mathrm{FH}$ is high at total cholesterol levels greater than $9.3 \mathrm{mmol} / 1$ or with low triglyceride levels [30], and those with raised triglycerides are less likely to have genetically confirmed FH. To further increase robustness of our findings, we performed sensitivity analyses with a subgroup of subjects with lower triglyceride levels and this demonstrated consistent findings for the association between CHD, stroke/TIA and PVD risk with FH.

While study size and design have enabled the risk of CVD outcomes in the identified population to be determined with considerable precision, we recognise some limitations. As with all clinical database analyses, findings are dependent on the quality of information entered. Although this is quality assured in practices contributing to CPRD [13], there will be incomplete recording of some lifestyle CVD risk factors, as well as some coded diagnoses and comorbidities. For instance, the nature of disease coding in the electronic health records did not specify if diagnosed FH subjects in this cohort were genetically confirmed. Genetic testing for FH is not yet widely available across the UK, so it is likely that diagnosis of FH by specialists were based on clinical criteria.

We also acknowledge that inadequate primary care assessment or documentation of premature CHD family history and other clinical features of FH such as premature corneal arcus and tendinous xanthomata [31,32] may have led to $\mathrm{FH}$ under-identification or misclassification. For instance, the prevalence of type 2 diabetes was slightly higher among FH subjects (2.2\%) than non-FH subjects (1.7\%). This may indicate that subjects with co-existing type 2 diabetes and CVD have been excluded, or may indicate potential misclassification of hypercholesterolaemia as FH rather than due to secondary causes such as diabetes. However, the prevalence is actually lower than prevalence of type 2 diabetes in genetically-confirmed $\mathrm{FH}$ subjects (5.9\%) in a recent EU registry study [33]. The selection criteria for non-FH subjects included cholesterol testing within six months of their matched $\mathrm{FH}$ cases, so these individuals represent a cohort of high-risk subjects who are being monitored with regular cholesterol testing. Furthermore, because of the overlap in distribution of lipid level in FH and non-FH subjects, a proportion of non-FH subjects may have cholesterol concentrations which are high enough to be compatible with an $\mathrm{FH}$ diagnosis, for example having a "polygenic" and not a monogenic cause [34]. As a consequence of these factors, this study may have in fact underestimated the CVD risk of FH compared to the general population.

Finally, data on adherence to lipid-lowering treatments is not available in routine electronic health records. Data on cholesterol levels during follow-up were also not collected in this study, so we were unable to ascertain the proportion of $\mathrm{FH}$ subjects on statins who were being treated to target. A recent UK study has shown variation in LDLcholesterol lowering in the general population of patients on statins, demonstrating that those patients not achieving LDL targets have increased risk of CVD [35]. We found that a higher proportion of diagnosed FH subjects were on lipid-lowering treatments compared with undiagnosed FH subjects, and the risk of CVD outcomes were much higher in undiagnosed subjects than those with documented diagnosis. We therefore speculate that the inflated CVD risk in undiagnosed $\mathrm{FH}$ subjects is due to sub-optimal management of these subjects. We also cannot rule out the probability that subjects with a known FH diagnosis are more likely to engage with intensive lifestyle modification and other CVD-risk lowering interventions than subjects without a known diagnosis.

\subsection{Clinical implications}

The findings of higher risk of stroke/TIA and PVD in addition to CHD risk, particularly in phenotypic undiagnosed FH subjects, underscore the vital importance of better and earlier identification of subjects with clinical FH. Primary care may be particularly well placed to do this in the general population. However, the finding that over the duration of the study, only $75 \%$ of the clinical FH patients in our cohort were on any lipid-lowering treatment, and only $38 \%$ of those on lipid-lowering treatment were on high-potency statins, is of serious concern and needs to be addressed. While this demonstrates that primary care practitioners are recognising and commencing treatment for patients with high cholesterol, it also highlights the fact that a majority of these $\mathrm{FH}$ subjects are probably not being referred to lipid clinics. These patients are not likely optimally managed with the level of statin treatment recommended by the NICE guidelines [8].

Knowledge of this increased cardiovascular disease burden, at a young age, should inform appropriate strategies for policy, practice and patient awareness. The current emphasis in prevention and management of FH in both primary and specialist care is to reduce increased risk of CHD $[8,36]$. Such care pathways should also focus on intensive lifestyle modification and treatment to reduce the risk of stroke/TIA and PVD.

\subsection{Conclusions}

Individuals with $\mathrm{FH}$ have been shown to have greatly increased risk of a range of CVD outcomes, including not only CHD but also stroke, TIA and PVD. This has important clinical implications and emphasises the need for improved case identification of clinically recognisable $\mathrm{FH}$ in the general population for targeted preventive intervention.

\section{Conflicts of interest}

NQ is a member of the National Institute for Health and Care Excellence (NICE) familial hypercholesterolaemia guideline development group (CG71). SW is a member of the Clinical Practice Research datalink Independent Scientific Advisory Committee (ISAC). The remaining authors have no competing interests.

\section{Financial support}

This study was supported by the Division of Primary care, University of Nottingham, and funded by the NIHR Health Technology Assessment, project number 15/134/02. BI's clinical academic 
lectureship is fully funded by the NIHR. SEH would like to acknowledge grants RG3008 and RG/08/014 from the British Heart Foundation, and the support of the UCLH NIHR BRC. The views expressed are those of the authors and not necessarily those of the NHS, the NIHR or the Department of Health \& Social Care.

\section{Author contributions}

BI led on the data management, conducted analyses, interpretation of findings, wrote the first draft and subsequent revisions. SW developed the study design and analysis methods, obtained ethical and study approvals to access the data, conducted the matching and extracted the study cohort. RA contributed to data cleaning, data management and variable coding. NQ and JK contributed to conceptualising the study design and methods, and provided primary care interpretation of findings. JLB provided statistical direction and advice on analysis methods. PR advised on refining study design and covariate selection. $\mathrm{SH}$ provided expert interpretation of study findings and formulated rationale for additional analyses. SW verified all analyses. SW, NQ, JK, $\mathrm{SH}, \mathrm{PR}, \mathrm{JLB}$ secured grant funding. All study authors have contributed to interpreting, revising, writing and finalising the final submission version of the manuscript.

\section{Acknowledgements}

The authors would like to thank members of the study team and steering committee of the Cascade FH study team involved with the NIHR funded Health Technology Assessment project 15/134/02.

\section{Appendix A. Supplementary data}

Supplementary data to this article can be found online at https:// doi.org/10.1016/j.atherosclerosis.2019.05.017.

\section{References}

[1] M.A. Austin, C.M. Hutter, R.L. Zimmern, S.E. Humphries, Genetic causes of monogenic heterozygous familial hypercholesterolemia: a HuGE prevalence review, Am. J. Epidemiol. 160 (2004) 407-420.

[2] B.G. Nordestgaard, M.J. Chapman, S.E. Humphries, H.N. Ginsberg, L. Masana, O.S. Descamps, et al., Familial hypercholesterolaemia is underdiagnosed and undertreated in the general population: guidance for clinicians to prevent coronary heart disease : consensus Statement of the European Atherosclerosis Society, Eur Heart J. 34 (2013) 3478-3490 08/15.

[3] L.E. Akioyamen, J. Genest, S.D. Shan, R.L. Reel, J.M. Albaum, A. Chu, et al., Estimating the prevalence of heterozygous familial hypercholesterolaemia: a systematic review and meta-analysis, BMJ Open 7 (Sep 1 2017) e016461.

[4] Risk of fatal coronary heart disease in familial hypercholesterolaemia. Scientific Steering Committee on behalf of the Simon Broome Register Group, BMJ Br. Med. J. (Clin. Res. Ed.) 303 (1991) 893-896.

[5] A. Neil, J. Cooper, J. Betteridge, N. Capps, I. McDowell, P. Durrington, et al., Reductions in all-cause, cancer, and coronary mortality in statin-treated patients with heterozygous familial hypercholesterolaemia: a prospective registry study, Eur. Heart J. 29 (Nov 2008) 2625-2633.

[6] Mortality in treated heterozygous familial hypercholesterolaemia: implications for clinical management. Scientific Steering Committee on behalf of the Simon Broome Register Group, Atherosclerosis 142 (Jan 1999) 105-112.

[7] J. Besseling, G.K. Hovingh, R. Huijgen, J.J.P. Kastelein, B.A. Hutten, Statins in familial hypercholesterolemia: consequences for coronary artery disease and all-cause mortality, J. Am. Coll. Cardiol. 68 (Jul 19 2016) 252-260.

[8] National Institute for Health and Care Excellence, Familial Hypercholesterolaemia: Identification and Management National Institute for Health and Care Excellence, NICE clinical guideline (CG71), 2008 Available: https://www.nice.org.uk/ guidance/cg71.

[9] R.R. Huxley, M.H. Hawkins, S.E. Humphries, F. Karpe, H.A. Neil, G. Simon Broome Familial Hyperlipidaemia Register, et al., Risk of fatal stroke in patients with treated familial hypercholesterolemia: a prospective registry study, Stroke 34 (Jan 2003) 22-25.

[10] S. Beheshti, C.M. Madsen, A. Varbo, M. Benn, B.G. Nordestgaard, Relationship of familial hypercholesterolemia and high low-density lipoprotein cholesterol to ischemic stroke, Circulation 138 (Aug 7 2018) 578-589.

[11] F. Emanuelsson, B.G. Nordestgaard, M. Benn, Familial hypercholesterolemia and risk of peripheral arterial disease and chronic kidney disease, J. Clin. Endocrinol. Metab. 103 (Dec 1 2018) 4491-4500.

[12] M. Kaste, P. Koivisto, Risk of brain infarction in familial hypercholesterolemia, Stroke 19 (Sep 1988) 1097-1100.

[13] E. Herrett, A.M. Gallagher, K. Bhaskaran, H. Forbes, R. Mathur, T. van Staa, et al., Data resource profile: clinical practice research datalink (CPRD), Int. J. Epidemiol. 44 (Jun 2015) 827-836.

[14] A.M. Perak, H. Ning, S.D. de Ferranti, H.C. Gooding, J.T. Wilkins, D.M. Lloyd-Jones, Long-term risk of atherosclerotic cardiovascular disease in us adults with the familial hypercholesterolemia phenotype, Circulation 134 (2016) 9-19.

[15] S. Hennessy, W.B. Bilker, J.A. Berlin, B.L. Strom, Factors influencing the optimal control-to-case ratio in matched case-control studies, Am. J. Epidemiol. 149 (Jan 15 1999) 195-197.

[16] P.H. Lee, Is a cutoff of $10 \%$ appropriate for the change-in-estimate criterion of confounder identification? J. Epidemiol. 24 (2014) 161-167 03/05.

[17] A.B. Pedersen, E.M. Mikkelsen, D. Cronin-Fenton, N.R. Kristensen, T.M. Pham, L. Pedersen, et al., Missing data and multiple imputation in clinical epidemiological research, Clin. Epidemiol. 9 (2017) 157-166.

[18] M. Futema, R.A. Whittall, A. Kiley, L.K. Steel, J.A. Cooper, E. Badmus, et al., Analysis of the frequency and spectrum of mutations recognised to cause familial hypercholesterolaemia in routine clinical practice in a UK specialist hospital lipid clinic, Atherosclerosis 229 (Jul 2013) 161-168.

[19] K. Haralambos, S.D. Whatley, R. Edwards, R. Gingell, D. Townsend, P. AshfieldWatt, et al., Clinical experience of scoring criteria for Familial Hypercholesterolaemia (FH) genetic testing in Wales, Atherosclerosis 240 (May 2015) 190-196.

[20] M.R. Law, N.J. Wald, A.R. Rudnicka, Quantifying effect of statins on low density lipoprotein cholesterol, ischaemic heart disease, and stroke: systematic review and meta-analysis, BMJ 326 (Jun 28 2003) 1423.

[21] L. Perez de Isla, R. Alonso, N. Mata, C. Fernandez-Perez, O. Muniz, J.L. Diaz-Diaz, et al., Predicting cardiovascular events in familial hypercholesterolemia: the SAFEHEART registry (Spanish familial hypercholesterolemia cohort study), Circulation 135 (May 30 2017) 2133-2144.

[22] L.J. Mundal, J. Igland, M.B. Veierod, K.B. Holven, L. Ose, R.M. Selmer, et al., Impact of age on excess risk of coronary heart disease in patients with familial hypercholesterolaemia, Heart 104 (Apr 5 2018:) 1600-1607.

[23] Risk of fatal coronary heart disease in familial hypercholesterolaemia. Scientific Steering Committee on behalf of the Simon Broome Register Group, BMJ 303 (Oct 12 1991) 893-896.

[24] J. Robson, I. Dostal, V. Madurasinghe, A. Sheikh, S. Hull, K. Boomla, et al., NHS Health Check comorbidity and management: an observational matched study in primary care, Br. J. Gen. Pract. 67 (Feb 2017) e86-e93.

[25] A. Zamora, L. Masana, M. Comas-Cufi, A. Vila, N. Plana, M. Garcia-Gil, et al., Familial hypercholesterolemia in a European Mediterranean population-Prevalence and clinical data from 2.5 million primary care patients, J Clin Lipidol 11 (Jul - Aug 2017) 1013-1022.

[26] M. Benn, G.F. Watts, A. Tybjaerg-Hansen, B.G. Nordestgaard, Familial hypercholesterolemia in the Danish general population: prevalence, coronary artery disease, and cholesterol-lowering medication, J. Clin. Endocrinol. Metab. 97 (Nov 2012) 3956-3964.

[27] B.C. Bansal, A.K. Sood, C.B. Bansal, Familial hyperlipidemia in stroke in the young, Stroke 17 (Nov-Dec 1986) 1142-1145.

[28] P.T. Kuo, J.F. Toole, J.A. Schaaf, A. Jones, A.C. Wilson, J.B. Kostis, et al., Extracranial carotid arterial disease in patients with familial hypercholesterolemia and coronary artery disease treated with colestipol and nicotinic acid, Stroke 18 (Jul-Aug 1987) 716-721.

[29] L.E. Akioyamen, J. Genest, A. Chu, H. Inibhunu, D.T. Ko, J.V. Tu, Risk factors for cardiovascular disease in heterozygous familial hypercholesterolemia: a systematic review and meta-analysis, J Clin Lipidol 13 (Jan - Feb 2019) 15-30.

[30] M. Futema, M. Kumari, C. Boustred, M. Kivimaki, S.E. Humphries, Would raising the total cholesterol diagnostic cut-off from $7.5 \mathrm{mmol} / \mathrm{L}$ to $9.3 \mathrm{mmol} / \mathrm{L}$ improve detection rate of patients with monogenic familial hypercholesterolaemia? Atherosclerosis 239 (2015) 295-298.

[31] P. Dhiman, J. Kai, L. Horsfall, K. Walters, N. Qureshi, Availability and quality of coronary heart disease family history in primary care medical records: implications for cardiovascular risk assessment, PLoS One 9 (2014) e81998.

[32] N. Qureshi, S.E. Humphries, M. Seed, P. Rowlands, R. Minhas, N.G.D. Group, Identification and management of familial hypercholesterolaemia: what does it mean to primary care? Br. J. Gen. Pract. 59 (Oct 2009) 773-776.

[33] E. Climent, S. Perez-Calahorra, V. Marco-Benedi, N. Plana, R. Sanchez, E. Ros, et al., Effect of LDL cholesterol, statins and presence of mutations on the prevalence of type 2 diabetes in heterozygous familial hypercholesterolemia, Sci. Rep. 7 (Jul 17 2017) 5596.

[34] M. Futema, S. Shah, J.A. Cooper, K. Li, R.A. Whittall, M. Sharifi, et al., Refinement of variant selection for the LDL-C genetic risk score in the diagnosis of the polygenic form of clinical Familial Hypercholesterolemia and replication in samples from six countries, Clin. Chem. 61 (11/20 2015) 231-238.

[35] R.K. Akyea, J. Kai, N. Qureshi, B. Iyen, S.F. Weng, Sub-optimal cholesterol response to initiation of statins and future risk of cardiovascular disease, Heart (2019 April 15), https://doi.org/10.1136/heartjnl-2018-314253.

[36] Public Health England, Familial Hypercholesterolaemia - Implementing a Systems Approach to Detection and Management, (2018). 